\title{
Ultranarrow-linewidth levitated nano-oscillator for testing dissipative wave-function collapse
}

\author{
A. Pontin ${ }^{(0)},{ }^{*}$ N. P. Bullier, M. Toroš $\odot$, and P. F. Barker ${ }^{\dagger}$ \\ Department of Physics \& Astronomy, University College London, Gower Street, London WC1E 6BT, United Kingdom
}

(Received 2 December 2019; accepted 27 May 2020; published 16 June 2020)

\begin{abstract}
Levitated nano-oscillators are promising platforms for testing fundamental physics and quantum mechanics in a new high mass regime. Levitation allows extreme isolation from the environment, reducing the decoherence processes that are crucial for these sensitive experiments. A fundamental property of any oscillator is its linewidth and mechanical quality factor $Q$. Narrow linewidths in the microhertz regime and mechanical $Q$ 's as high as $10^{12}$ have been predicted for levitated systems. The insufficient long-term stability of these oscillators has prevented direct measurement in high vacuum. Here we report on the measurement of an ultranarrow linewidth levitated nano-oscillator, whose width of $81 \pm 23 \mu \mathrm{Hz}$ is only limited by residual gas pressure at high vacuum despite residual variations of the trapping potential. This narrow linewidth allows us to put new experimental bounds on dissipative models of wave-function collapse including continuous spontaneous localization and Diósi-Penrose and illustrates its utility for future precision experiments that aim to test the macroscopic limits of quantum mechanics.
\end{abstract}

DOI: 10.1103/PhysRevResearch.2.023349

\section{INTRODUCTION}

Levitated oscillators formed by trapping neutral and charged nanoparticles in optical [1-4], electric [5-8], or magnetic fields $[9,10]$ are unlike any other optomechanical system in that the oscillator's properties can be widely tuned by control of the levitating fields. The fields can even be turned-off, offering low noise, field free, measurements for short periods of time [11,12]. These properties make them an attractive platform for exploring the foundations of quantum mechanics and fundamental physics in a previously unexplored regime [12-16]. A fundamental property of any oscillator is its linewidth and mechanical quality factor $Q$. Narrow linewidths in the submicrohertz range and quality factors has high as $10^{12}$ have been predicted for optical levitated systems [2]. However, the poor stability of these oscillators over long periods, coupled with their tendency to operate in anharmonic/nonlinear regimes has prevented direct measurement of the predicted narrow linewidths in high vacuum. For many levitated systems, the measured linewidths deviate from those predicted even at moderate vacuum in the $10^{-4}-10^{-5}$ mbar range $[4,17,18]$. The reduction of noise introduced by the levitating fields is a key challenge for achieving a stable oscillator. A fundamental limiting noise for optical levitation is the recoil of photons from the levitation laser itself [19], while internal heating via absorption of laser light leads to motional heating [20]. In contrast, a charged nanoparticle that

\footnotetext{
*a.pontin@ucl.ac.uk

†p.barker@ucl.ac.uk
}

Published by the American Physical Society under the terms of the Creative Commons Attribution 4.0 International license. Further distribution of this work must maintain attribution to the author(s) and the published article's title, journal citation, and DOI. is levitated using electrodynamic fields within a Paul trap, is an attractive levitated system as it is free from recoil induced heating $[5,6]$ as only low light intensities are required for detection. In addition, large well depths in excess of $1 \mathrm{eV}$ (up to $10^{6} \mathrm{~K}$ ) allow operation of the trap in the harmonic regime, even for temperatures exceeding $300 \mathrm{~K}$. We have recently demonstrated [21] that the charge on nanosphere in a Paul trap is stable over many weeks of measurement allowing stable oscillation frequencies limited only by the noise and drifts in the applied electric fields and environmental disturbances.

Here we report on a levitated nanoparticle oscillator operating at pressures down to $10^{-7} \mathrm{mbar}$ in a room temperature environment. We outline the application of a phase sensitive detection method that allows us to remove the small residual variations in the trapping potential over the very long timescales required to measure an ultralow linewidth of $81 \pm 23 \mu \mathrm{Hz}$. This is to the best of our knowledge, the narrowest linewidth measured for a mechanical oscillator and paves the way for future precision experiments using levitated systems. We also characterize the important noise sources for this oscillator and outline a means to achieve even lower linewidth measurements and higher $Q$ experiments for future experiments to test the macroscopic limits of quantum mechanics. Finally, we use our current oscillator to place new bounds on two wave-function collapse models in their dissipative variant, namely, continuous spontaneous localization and Diósi-Penrose [22-28] demonstrating its utility for future precision experiments that aim to test the macroscopic limits of quantum mechanics $[29,30]$.

\section{EXPERIMENTAL SETUP AND LINEWIDTH MEASURMENT}

We create our levitated nano-oscillator by trapping commercial silica nanospheres of radius $230 \mathrm{~nm}$ in high vacuum within a linear Paul trap [21] shown schematically in Fig. 1. 


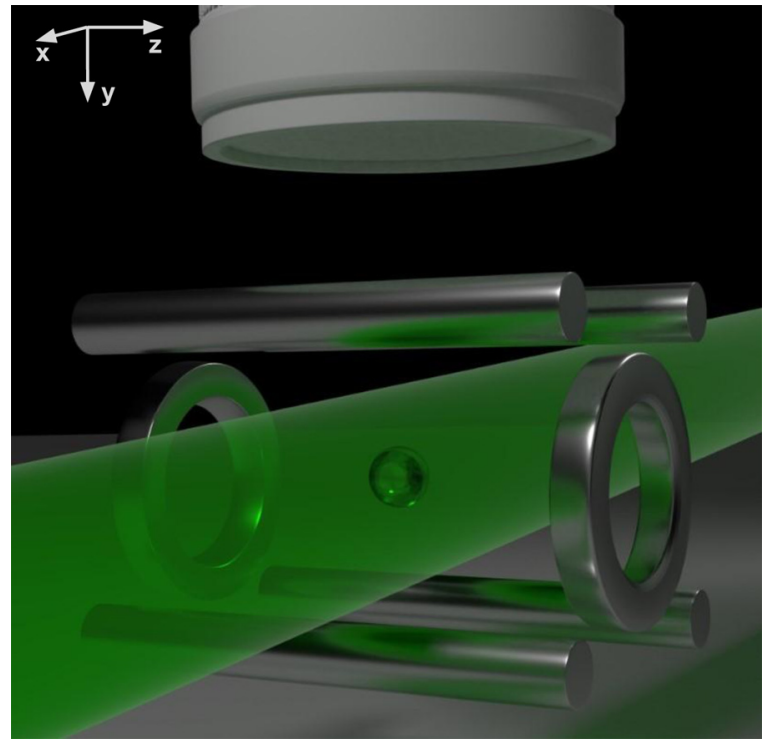

FIG. 1. Schematic view of the experiment. A silica nanoparticle is levitated in a linear Paul trap at high vacuum. The motion is detected by illuminating the particle with a wide green laser field and imaging the light scattered at $90^{\circ}$.

It consists of four stainless steel rods $(0.5 \mathrm{~mm}$ diameter $)$ that provide transverse confinement and two, coaxial ring electrodes that provide axial confinement. The typical chargeto-mass ratio we levitate is between 0.05 and $2 \mathrm{C} / \mathrm{kg}$. The position of the particle as a function of time is determined from fast imaging of the particle illuminated by a low intensity laser beam $\left(20 \mathrm{~W} \mathrm{~cm}^{-2}\right)$. This low intensity guarantees that the particle is not perturbed and that the internal temperature remains close to $300 \mathrm{~K}$ [20]. Additionally, shot noise recoil heating from the probe beam is also negligible.

The oscillator dynamics are described by a set of Mathieu equations. The resulting trajectories are stable if the $a$ and $q$ parameters fall into the well known stability region [31]. Under the additional condition of $q \lesssim 0.4$, a pseudopotential approximation provides a very good description of the particle motion. In this case, the trapping potential is harmonic with frequencies along the $i$ axis given by $\omega_{i} \cong \omega_{d} / 2 \sqrt{a_{i}+q_{i}^{2} / 2}$. Collisions with the residual gas result in a damped harmonic motion driven by a stochastic force $F_{\text {th }}$ with power spectral density (PSD) $S_{F_{\mathrm{th}} F_{\mathrm{th}}}=2 k_{B} T m \gamma$. An example of such thermal motion obtained with our detection scheme is shown in Fig. 2. At the lowest pressure, other noise sources such as voltage noise on the trap electrodes or ambient displacement noise become important and must be controlled for stable operation.

One of the main advantages of the levitated platform lies in the possibility of achieving a very small coupling between the oscillator and the thermal bath. Assuming a particle internal temperature at equilibrium with the background gas at temperature $T$, inelastic collisions provide a damping rate given by $\gamma=4 \pi m_{g} R^{2} v_{t} P_{g} /\left(3 k_{B} T m\right)(1+\pi / 8)[32,33]$, where $v_{t}=$ $\sqrt{8 k_{B} T /(\pi m)}$ is the gas thermal velocity, $m_{g}$ the molecular mass and $P_{g}$ is the residual gas pressure. At relatively high pressures, the damping rate can be accurately measured, even for large particles (i.e., $\sim 1-30 \mu \mathrm{m}$ ), however, as the pressure

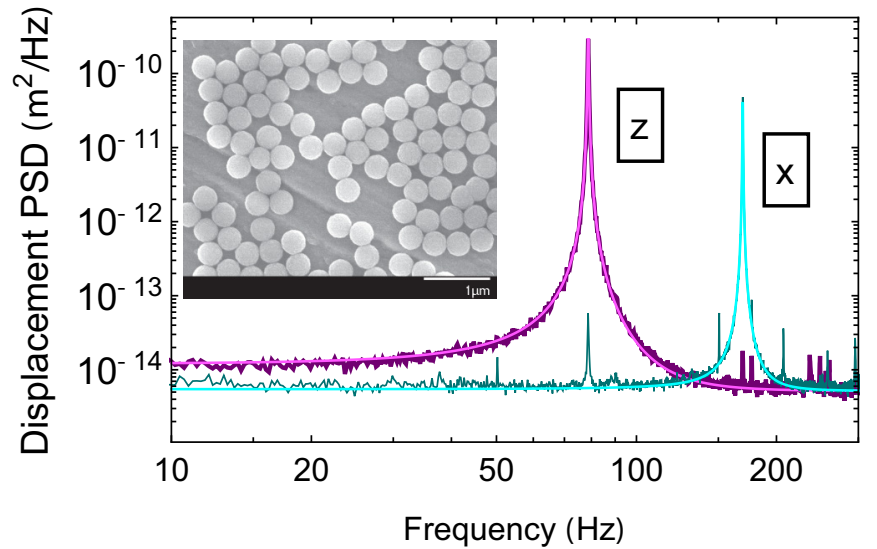

FIG. 2. Displacement PSD of a trapped nanoparticle along the two degrees of freedom monitored at a pressure $P_{g}=10^{-3} \mathrm{mbar}$ for a particle of $231 \mathrm{~nm}$ radius; also shown are fits with the mechanical susceptibility (see Methods). (Inset) Scanning electron microscope image of the nanoparticles used.

is reduced, it becomes increasingly difficult to perform accurate direct measurements. There are many examples in the literature demonstrating a saturation of the linewidth for levitated systems $[4,17]$. Spectral estimation requires continuous monitoring for timescales much longer than the correlation time $(2 / \gamma)$ with the implicit requirement that the stability of the trap frequency is far better than the linewidth, i.e., $\delta \omega_{i} \ll \gamma$. On the other hand, for long correlation times, timeresolved techniques are usually preferred [34]. However, this approach requires driving the particle to large amplitudes in order to achieve a good signal-to-noise ratio which could lead to particle loss and, more often, to the exploration of highly nonlinear regions of the trap potential.

We remove the problems related to the trapping potential stability by implementing a numerical phase sensitive detection. To this end, it is convenient to move to a frame rotating at the mechanical frequency $\omega_{i}$. The motion $u_{i}(t)$ along any axis $i$ can be decomposed into two quadratures $X_{i}(t)$ and $Y_{i}(t)$ according to $u_{i}(t)=X_{i}(t) \cos \left(\omega_{i} t\right)+Y_{i}(t) \sin \left(\omega_{i} t\right)$. This approximation neglects fast rotating terms at $2 \omega_{i}$, however, it is well known that this is a very good approximation for high- $Q$ oscillators for which changes of the quadratures amplitude happen adiabatically with respect to the potential. To simplify the notation, we consider only one degree of freedom and denote its resonance frequency with $\omega_{o}$. If $\gamma \ll \omega_{o}$, the dynamical equations for the slowly varying quadratures are [35]

$$
\begin{aligned}
& \dot{X}+\frac{\gamma}{2} X=\frac{1}{m \omega_{o}} f^{(1)}, \\
& \dot{Y}+\frac{\gamma}{2} Y=\frac{1}{m \omega_{o}} f^{(2)},
\end{aligned}
$$

where $f^{(k)}$ are stochastic force terms with autocorrelation functions $\left\langle f^{(k)}(t) f^{(j)}\left(t^{\prime}\right)\right\rangle=\delta_{k j} \delta\left(t-t^{\prime}\right) S_{\mathrm{FF}} / 2$, assuming a delta-correlated force noise $F$, with PSD $S_{F}$, driving the oscillator. The spectra of the two quadratures are then $S_{\mathrm{XX}}(\omega)=$ $S_{\mathrm{YY}}(\omega)=\frac{S_{\mathrm{FF}}}{2\left(m w_{o}\right)^{2}} \frac{1}{\omega^{2}+\gamma^{2} / 4}$. Experimentally, these PDSs are obtained by implementing a numerical lock-in amplifier, where 
we demodulate the displacement signal $u_{o}(t)$ at a frequency $w_{\mathrm{LO}}$ and filter out the fast varying components at $2 \omega_{o}$. The two quadratures are still affected by changes of the trap frequencies or by a frequency difference between $\omega_{o}$ and the reference rotation $\omega_{\mathrm{LO}}$; if $\omega_{o}=\omega_{\mathrm{LO}}+\delta \omega$ their spectrum will be given by a low-frequency Lorentzian peak centered at $\delta \omega$. Of course, any time dependence of $\omega_{o}$ will be mapped on $\delta \omega$ leading to a broadened peak and a linewidth estimation that is incorrect or impossible.

However, frequency drifts or offsets are removed if we look at the amplitude quadrature $R=\sqrt{X^{2}+Y^{2}}$. This can be understood with a simple heuristic argument. Let us consider an almost sinusoidal signal $u(t)=A(t) \cos \left\{\left[\omega_{o}+\delta \omega(t)\right] t\right\}$ with a slowly varying amplitude and central frequency. After demodulation and low pass filtering the two quadratures will be

$$
\begin{aligned}
& X(t)=\frac{1}{2} A(t) \cos [\delta \omega(t) t], \\
& Y(t)=-\frac{1}{2} A(t) \sin [\delta \omega(t) t]
\end{aligned}
$$

as long as $|\delta \omega(t)| \ll \omega_{o}$, i.e., changes of the central frequency are adiabatic. The amplitude quadrature is then given by $R(t)=\sqrt{X(t)^{2}+Y(t)^{2}}=A(t) / 2$, which is unaffected by the time dependence of $\delta \omega(t)$. Obtaining an analytical expression for the amplitude quadrature spectrum $S_{\mathrm{RR}}(\omega)$ is not trivial, however, neglecting the time dependence of $\omega_{o}$, it is possible to evaluate analytically the PSD of $S_{R^{2} R^{2}}(\omega)$ which is given by [36]

$$
S_{R^{2} R^{2}}(\omega)=\frac{8}{\gamma} \frac{1}{\omega^{2}+\gamma^{2}}\left(\frac{S_{F}}{2 m^{2} w_{o}^{2}}\right)^{2} .
$$

Importantly, Eq. (3) holds even for an oscillator with a slowly varying resonance as can be shown numerically and as we demonstrate experimentally in the following.

\section{DISCUSSION}

Here we present data acquired by monitoring a single nanoparticle of mass $m=9.6 \pm 0.4 \pm 0.9 \times 10^{-17} \mathrm{~kg}$ [37] obtained by inducing a change of the total charge by one elementary charge by and measuring the resulting shift in the trap frequency [21]. Assuming a nominal density of $\rho=$ $1850 \mathrm{~kg} / \mathrm{m}^{3}$ this corresponds to a radius of $r=231 \pm 7 \mathrm{~nm}$.

The frequency stability in our trap is of the order of $\delta \omega_{\max } / \omega_{o}=0.003$ over a timescale of an hour. This is dominated by thermal drifts in the electronics that supply the electrode voltages and as well as to slowly changing stray fields. At the lowest pressures, this frequency drift is much larger than the expected linewidth. Furthermore, the behavior along the two axis are significantly different. Along the trap axis $(z$ axis in Fig. 1) the resonance goes through a slow smooth drift, while in the orthogonal direction ( $x$ axis) there is an additional periodic modulation of $\delta \omega_{x} / 2 \pi=0.25 \mathrm{~Hz}$ with period of roughly an hour. This can clearly be seen in Fig. 3 where we show the displacement PSD along both axes at two different pressures $\left[P_{g}=10^{-4}\right.$ (red curves) and $2 \times 10^{-5}$ mbar (blue curves)]. The effect of the frequency drift/modulation in the $x$ axis is evident by the flattening of the spectral profile. Even in the $z$ axis, where the PSD is not

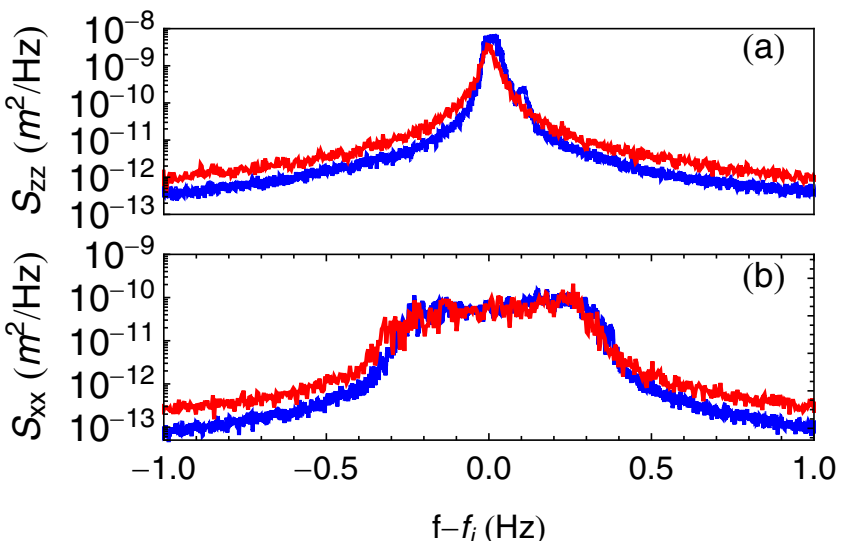

FIG. 3. Displacement PSD of a trapped nanoparticle along the two degrees of freedom monitored. The PSDs refer to two pressures $P_{g}=10^{-4}$ (red) and $2 \times 10^{-5} \mathrm{mbar}$ (blue). The linewidth measured exploiting the $R^{2}$ spectra are respectively $28.5 \pm 0.7$ and $7.5 \pm$ $0.5 \mathrm{mHz}$.

strongly perturbed, the conventional spectral approach yields a reliable linewidth measurement only at higher pressures.

In contrast, the linewidth can be determined from the PSD of $R^{2}$ as shown in Figs. 4(a)-4(c) for different pressures along the $x$ axis. Also shown is a fit to the data using Eq. 3. The linewidth follows the expected behavior from gas collisions very well despite the frequency modulation that was clearly visible in the conventional PSDs in Fig. 3(b). We point out that the modulation in the secular frequency $\delta \omega_{x}$, at the lowest pressure, is 2500 times larger than the linewidth $\gamma$, illustrating the utility of this method for extracting narrow linewidths in the presence of slow, large modulation and drifts in the trap frequency. To test the accuracy of this approach we fit the much wider high pressure measurements performed with the standard spectral method and compare them to the linewidths obtained from the $R^{2}$ spectra. We find perfect consistency between the two methods (see Methods). The linewidths as a function of pressure, averaged between the two degrees of freedom, are fitted with a simple line, i.e., $\gamma=\gamma_{\mathrm{exc}}+k P_{g}$, to allow the estimation of a possible excess damping $\gamma_{\text {exc }}$. The fit is shown in Fig. 5 along with the 95\% confidence bands. With the same confidence level we find $\gamma_{\mathrm{exc}} / 2 \pi=18 \pm 30 \mu \mathrm{Hz}$, which is consistent with zero confirming that only gas damping is affecting the particle dynamic. The minimum measured linewidth is the ultralow value of $81 \pm 23 \mu \mathrm{Hz}$.

We show in Figs. 4(d)-4(f) histograms of the amplitude quadrature at different pressures. This is the well known Rayleigh distribution $\mu\left(r ; \sigma_{i}\right)=r / \sigma_{i}^{2} e^{-r^{2} / \sigma_{i}^{2}}$ with mean and variance given by $\langle r\rangle=\sqrt{\pi / 2} \sigma_{i}$ and $\sigma_{\mu}^{2}=(4-\pi) \sigma_{i}^{2} / 2$, respectively. If the motion along the $i$-axis is in thermal equilibrium with the environment we simply have $\sigma_{i}^{2}=k_{B} T / m \omega_{i}^{2}$ which is the usual thermal variance for an harmonic oscillator. The three data sets have a similar total observation time of the order of one day. This implies that the number of statistically independent points significantly drops when the pressure is reduced to the minimum value. The sample size goes from approximately 10000 at $P_{g}=9 \times 10^{-5}$ mbar to 100 at $P_{g}=3 \times 10^{-7}$ mbar as can clearly be observed in Figs. 4(d) and 4(f). We exploit the properties of the Rayleigh 
(a)

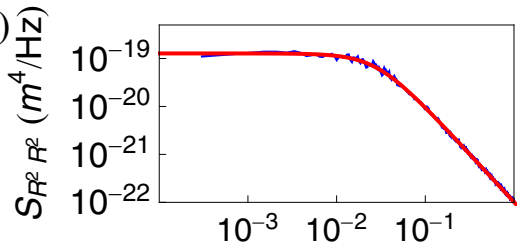

Frequency $(\mathrm{Hz})$

(d)

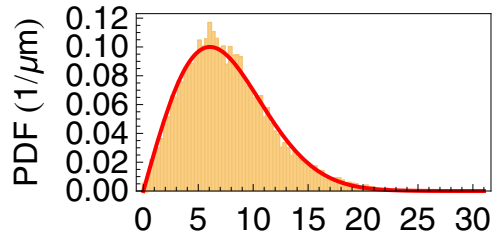

$\mathrm{R}(\mu \mathrm{m})$ (b)

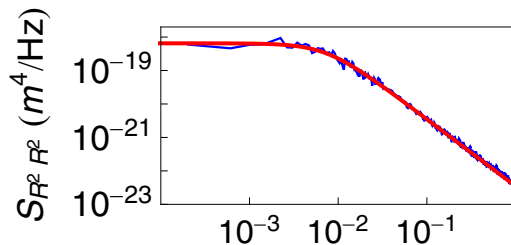

Frequency $(\mathrm{Hz})$

(e)

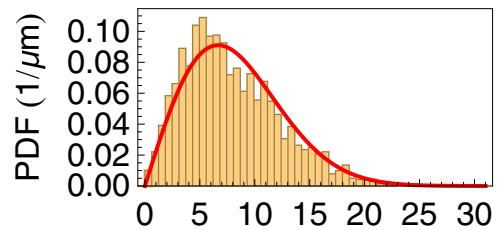

$\mathrm{R}(\mu \mathrm{m})$ (c)

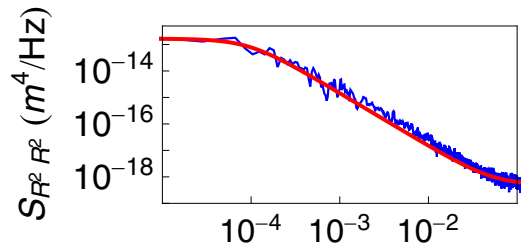

Frequency $(\mathrm{Hz})$

(f)

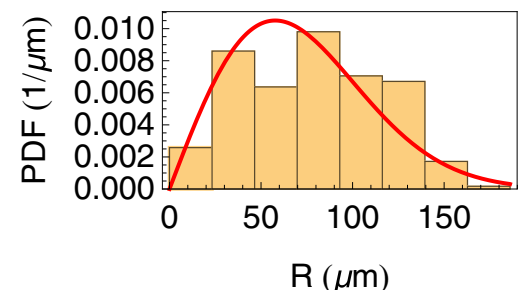

FIG. 4. [(a), (b), and (c)] PSD of the square amplitude quadrature for the motion along the $x$ axis along with a fit following Eq. (3) for different pressures $P_{g}=9 \times 10^{-5}, 2 \times 10^{-5}$, and $3 \times 10^{-7}$ mbar, respectively. [(d)-(f)] Probability density functions (PDF) of the amplitude quadrature $R$ for the same data sets along with the corresponding Rayleigh distribution. Each data set corresponds to a continuous acquisition close to one day.

distribution to verify that the sample size is big enough to be representative of the distribution. The first two moments allow an estimation of the motional variance which can be compared with each other. Specifically, at $P_{g}=9 \times 10^{-5}$ mbar we have $\Delta \sigma_{i} /\left\langle\sigma_{i}\right\rangle=0.005$, while at $P_{g}=3 \times 10^{-7} \mathrm{mbar}$, we find $\Delta \sigma_{i} /\left\langle\sigma_{i}\right\rangle=0.08$ which is in line with what is expected. Indeed, the relative difference of the two estimators should scale as the square root of the sample size.

It is clear, from both the PSD spectra and the Rayleigh distributions of Fig. 4, that the variance of the stochastic motion increases as the pressure is reduced. This behavior is also shown as an increase in the effective temperature as the pressure is reduced in Fig. 6. Here we plot the effective temperature along both trap axes as a function of pressure. This calibration does not rely on the assumption of thermal equilibrium at room temperature but exploits the absolute calibration available from using the camera [38]. For the high

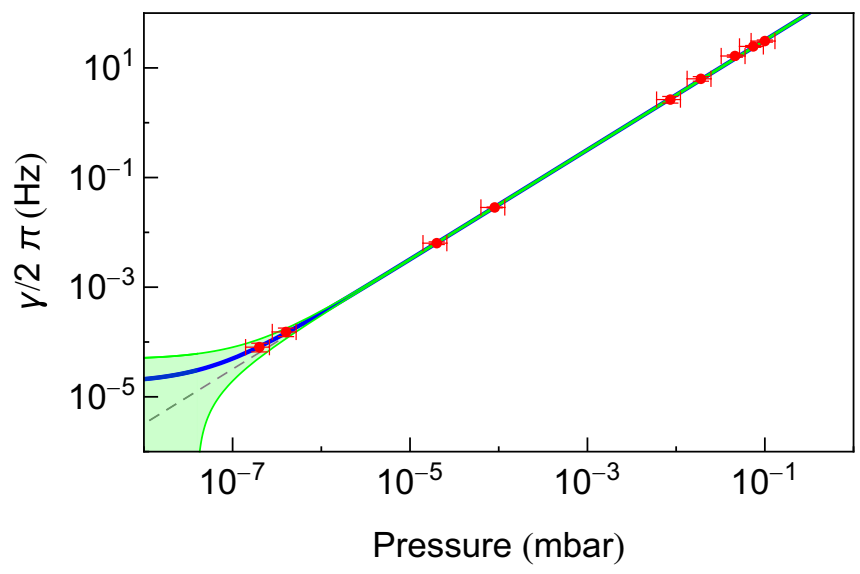

FIG. 5. Linewidths averaged over the two degrees of freedom measured and with a linear fit (blue) and $95 \%$ confidence bands (green). Also shown is the fit assuming no excess damping (dashed grey). pressures $\left(P_{g}>10^{-3} \mathrm{mbar}\right)$, the mean effective temperature is $\langle T\rangle=293 \pm 3 \pm 25 \mathrm{~K}$, where the systematic error comes from the uncertainty in the mass measurement. The excess force noise leads to an increase in effective temperature that varies inversely with pressure. A fit to the average temperature on the two axes gives $T_{\text {eff }}=293\left(1+7.3 \times 10^{-6} / P_{g}\right)$. We obtain a value for the excess force noise from the PSD of $S_{\mathrm{ff}} \simeq 1 \times 10^{-38} \mathrm{~N}^{2} / \mathrm{Hz}$ (heating rate of $240 \mathrm{MHz}$ ). For the nanoparticle illuminated by the detection beam, the back action due to photon recoil is approximately $4 \times 10^{-43} \mathrm{~N}^{2} / \mathrm{Hz}$ $(9 \mathrm{kHz})$ which is insignificant compared to the thermal noise of $10^{-40} \mathrm{~N}^{2} / \mathrm{Hz}(3 \mathrm{MHz})$ at $10^{-7}$ mbar. Assuming the excess noise that we observe is dominated by voltage noise, we estimate its value to be $\sqrt{S_{\mathrm{VV}}} \simeq 10 \mu \mathrm{V} / \sqrt{\mathrm{Hz}}$, which is consistent

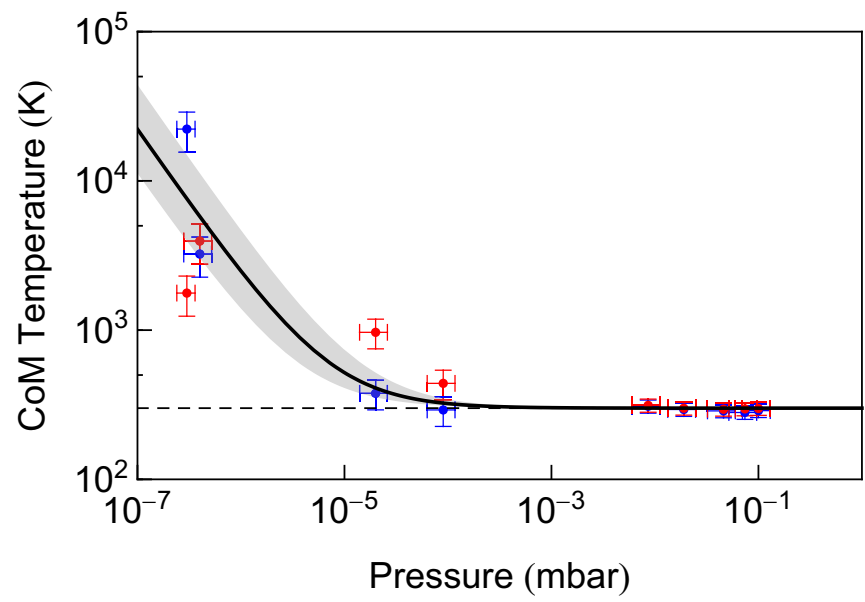

FIG. 6. Centre of mass (CoM) effective temperature as a function of pressure along both $x$ (blue) and $z$ (red) axes; the average between the two degrees of freedom is fitted assuming a $1 / P_{g}$ contribution that would rise from a white nonthermal force noise. A $3 \mathrm{~dB}$ temperature increase occurs at $P_{g}=7.3 \times 10^{-6}$ mbar. Dashed gray line marks the measured room temperature of $293 \mathrm{~K}$. 
with the measured voltage noise. This is determined from $S_{\mathrm{ff}}=\left(n_{\mathrm{ch}} e\right)^{2} S_{\mathrm{VV}} / D^{2}$, where $D=2.3 \mathrm{~mm}$ is the characteristic distance related to the AC electrode geometry (see Methods) and $n_{\mathrm{ch}} \simeq 80$ is the number of charges on the nanoparticle.

Our nanoscale oscillator, with a $Q$ of $1.5 \times 10^{6}$, compares well with the highest $Q$ factors ever reported for relatively low-frequency oscillators, particularly for operation at room temperature. On a similar experimental system, a $Q$ factor of $1.5 \times 10^{5}$ has been previously demonstrated [34]. For clamped oscillators, the most notable are balanced torsional oscillators (i.e., QPO) $[39,40]$ where $Q$ 's of almost $10^{6}$ are reached for higher oscillation frequencies of a few kilohertz. A singlecrystal silicon oscillator has demonstrated a linewidth of $\sim 500 \mu \mathrm{Hz}$ at $300 \mathrm{~K}$ [41] and a $Q$ of $4 \times 10^{7}$. For all clamped systems, however, only a very limited number of normal modes have such high $Q$ factors. This is in stark contrast with the levitated case described here where all three translational degrees of freedom of the oscillator experience the same dissipative forces, while the internal modes are completely decoupled as the lowest modes have frequencies greater than $\mathrm{GHz}[42]$.

\section{DISSIPATIVE COLLAPSE MODELS}

We now describe the use of our narrow linewidth oscillator, coupled with our ability to predict the simple variation of damping with pressure, to put new limits on two dissipative models for wave-function collapse. This includes the dissipative continuous spontaneous localization (CSL) [43] and the dissipative Diósi-Penrose (DP) [27,28,44] models. Recently, a microscale oscillator cooled to $20 \mathrm{mK}$ has been used to place stringent constraints on the CSL model $[45,46]$. Its effect on an oscillator is an additional stochastic force noise which has a white spectral density $S_{\mathrm{CSL}}=\hbar^{2} \eta_{\mathrm{CSL}}$ that induces a collapse of the wave function. This is characterized by a collapse strength $\eta_{\text {CSL }}$ (see methods) which depends on the geometry and density of the oscillator and upon two parameters, namely, the collapse rate $\lambda$ and a characteristic length $r_{C}$. The conventional CSL model is not energy conserving and a dissipative version (dCSL) [25] has been proposed which removes the energy divergence due to the standard CSL force noise. The additional dissipation determines a finite equilibrium temperature for any given system and an additional parameter $T_{\mathrm{dCSL}}$ is introduced which characterizes the temperature of the collapse noise. In the dCSL framework, the force noise is no longer white and for a nanoparticle it has a spectral density [22] $S_{\mathrm{dCSL}}(\omega)=\hbar^{2} \eta_{\mathrm{dCSL}}\left[1+\kappa^{2} m^{2}\left(\gamma_{t}^{2}+\right.\right.$ $\left.\left.\omega^{2}\right)\right]$, where $\gamma_{t}=\gamma+\gamma_{\mathrm{dCSL}}$ is the total damping rate and $\kappa=\gamma_{\mathrm{dCSL}} / 2 \hbar \eta_{\mathrm{dCSL}}$. In the limit of an infinite temperature the standard CSL is recovered.

The DP model has a structure quite similar to the CSL with the main difference being that the scale of the collapse strength is set by the gravitational constant $G$, thus introducing a stronger connection to gravity for the collapse mechanism. The standard DP model is also not energy conserving, and a dissipative version has been proposed [26]. Since the collapse strength is fixed, the dissipative DP (dDP) model is characterized by only two parameters, namely, the temperature of the collapse noise $T_{\mathrm{dDP}}$ and a cutoff distance $R_{0}$, which plays a similar role as $r_{C}$ in the dCSL model.
The CSL and DP family of models usually discussed in the literature predict that the collapse strength for the center-ofmass dynamics depends on the intensive and extensive properties of the bulk material, such as composition and shape. This is the version we consider for dCSL. On the other hand, we can also consider the dDP model in the single-particle version, which depends only on the total mass of the system, but not on any other property of the system (see Methods). In the following, we consider this latter version of the dDP model, while our experiment does not put significant bounds on the former one.

For both the dCSL and dDP models, the force noise sensitivity required to place significant bounds is quite far from what can be achieved in our current experiment. Indeed, here we exploit a new and completely different approach which compares the theoretical predictions for the damping due to the collapse mechanisms to our measurements of extremely narrow linewidth. This allows us to place significant constraints on the dCSL and dDP models. For the levitated oscillator, the damping rate is well defined by the collisions with the surrounding gas and has a linear dependence with pressure. This allows us to extrapolate its value at zero pressure with any offset potentially due to the collapse process. We find an upper limit for the damping rate due to the collapse mechanisms of $\gamma_{\mathrm{cm}} / 2 \pi \leqslant 48 \mu \mathrm{Hz}$ at the $95 \%$ confidence level which is compared to the predictions from the models.

Exploiting this upper limit we derive new bounds for dCSL which are shown in Fig. 7(a). These new bounds assume different dCSL temperatures. When $T_{\mathrm{dCSL}}=1 \mathrm{~K}$ our bounds are comparable to existing ones [47]. However, when $T_{\mathrm{dCSL}}$ is reduced to $10^{-7} \mathrm{~K}$ [22] the excluded parameter space is significantly increased reaching a minimum collapse $\lambda \simeq 10^{-14}$ $\mathrm{s}^{-1}$ for an $r_{C}=1.5 \mu \mathrm{m}$. Remarkably, for these values the force noise sensitivity required to place an equivalent bound on both CSL and dCSL is $\sim 10^{-50} \mathrm{~N}^{2} / \mathrm{Hz}$ which would be achievable only for a thermal particle at a pressure of $\sim 10^{-15}$ mbar (assuming the same particle size).

The excluded parameter space for the dDP model in the single-particle version is shown in Fig. 7(b). The value for $R_{0}$ originally proposed by Diósi is excluded on an extremely wide temperature range from $10^{-10}$ to $10^{10} \mathrm{~K}$, while at the GRW value, the exclusion is limited to very unrealistic $T_{\mathrm{DP}}$ values. A value considered reasonable is the cosmic microwave background (CMB) temperature [22]; in this case, the excluded $R_{0}$ goes from $10^{-18} \mathrm{~m}$ to $10^{-12} \mathrm{~m}$. Interestingly, the excluded region has no upper bound and extends to $T_{\infty}$ for increasingly smaller values of $R_{0}$.

\section{CONCLUSIONS}

We have demonstrated that a narrow linewidth, high- $Q$, levitated nano-oscillator can be created by trapping nanoparticles in the Paul trap. We show that, even in the presence of voltage noise from the electronics, the trap is stable enough to measure a record low mechanical linewidth of $\sim 80 \mu \mathrm{Hz}$ at room temperature. This compares well with other high$Q$ low-frequency oscillators that typically achieve similar performances at cryogenic temperatures. Using the narrow linewidth of this oscillator we place new constraints on two dissipative models for wave-function collapse, namely, the 
(a)

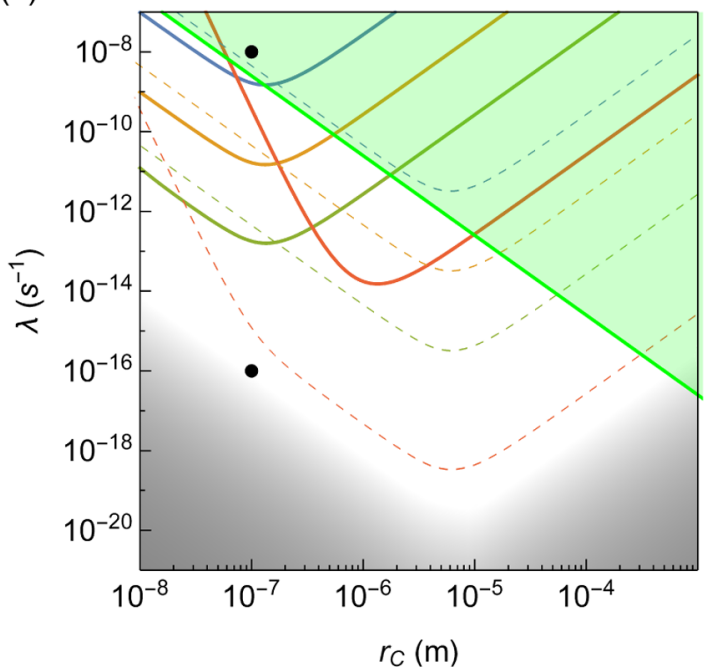

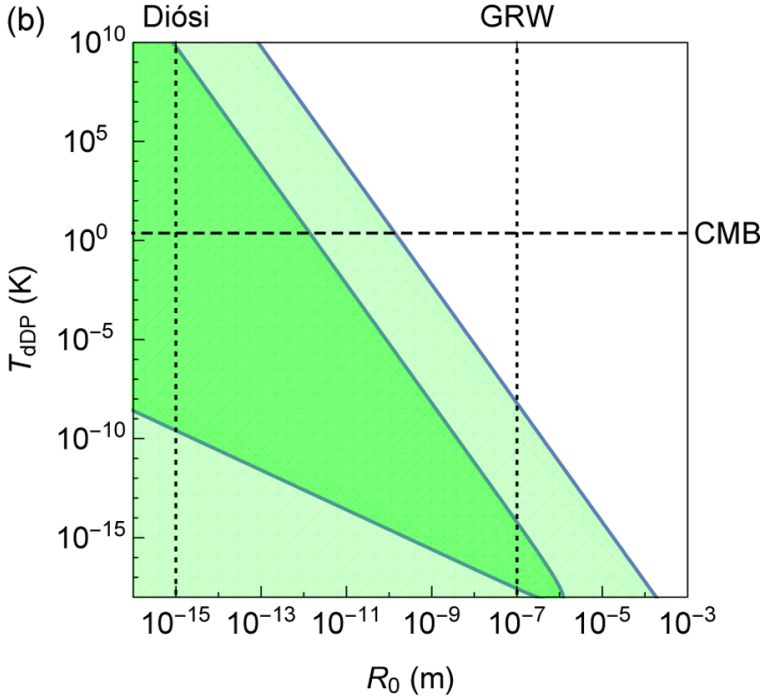

FIG. 7. Experimental bounds on dissipative collapse models. (a) Exclusion region for the dCSL parameters $\lambda$ and $r_{C}$. Continuous lines: upper bounds from current linewidth measurements for a $231 \mathrm{~nm}$ radius silica particle at $3 \times 10^{-7}$ mbar for different values of $T_{\mathrm{dCSL}}$ : 1 (blue), $10^{-2}$ (yellow), $10^{-4}$ (green), and $10^{-7} \mathrm{~K}$ (red). The dashed lines are the expected improved bounds from future measurements on a $10 \mu \mathrm{m}$ radius silica particle; same color code for $T_{\mathrm{CSL}}$. The green region corresponds to upper bound from LISA pathfinder [47-49] and the grey region is the lower bound from theoretical arguments [50,51]. The black markers refer to the Adler [52,53] (top) and GRW [43] (bottom) values. (b) Exclusion region for the single-particle dDP model. The darker green corresponds to the bounds from the current experiment. The lighter green region is the expected improved bounds from future measurements on a $10 \mu \mathrm{m}$ radius silica particle. We chose to limit the temperature to the lowest conjectured value of $10^{-18} \mathrm{~K}$ associated with Hawking radiation from a supermassive black hole [54,55]. CMB marks the cosmic microwave background temperature.

continuous spontaneous localization and the Diósi-Penrose in the single-particle version. By utilising lower noise electronics, and a larger mass oscillator which can be incorporated in this system, we expect to be able to provide even more stringent limits on standard and dissipative collapse models in the future. Considering a $10 \mu \mathrm{m}$ radius silica particle, and measuring a linewidth an order of magnitude smaller than measured here, would have a significant impact. In the case of the dCSL it would allow us to almost entirely exclude the $\lambda-r_{C}$ parameters subspace for the lowest $T_{\mathrm{dCSL}}$ [50,51]. In the case of the $\mathrm{dDP}$, we could exclude $R_{0}$ values almost two orders of magnitude larger at the CMB temperature. Achieving these objectives will require an improvement of the current electronics but nothing beyond the current state of the art. Furthermore, particular care might be necessary in order to mitigate the effect of vibrational noise. Finally, if such a particle is thermal at $P_{g} \sim 10^{-10}$ mbar our system would allow us to probe the standard CSL for collapse rates approaching $10^{-13} \mathrm{~s}^{-1}$ at a characteristic length of $\sim 6 \mu \mathrm{m}$ thus extending well beyond the current bounds.

\section{ACKNOWLEDGMENTS}

The authors acknowledge funding from the EPSRC Grant No. EP/N031105/1 and the H2020-EU.1.2.1 TEQ project Grant agreement ID: 766900. A.P. has received funding from the European Union's Horizon 2020 research and innovation programme under the Marie Sklodowska-Curie Grant Agreement ID: 749709 a N.P.B. acknowledges funding from the EPSRC Grant No. EP/L015242/1.

\section{APPENDIX A: DYNAMICS IN A LINEAR PAUL TRAP}

The linear Paul trap consists of four electrodes shown in Fig. 1. Along one diagonal the electrodes are grounded and on the other two they are driven by an AC electric field which provides a trapping potential in the plane perpendicular to the rods axis. To generate $3 \mathrm{D}$ confinement, two additional ring end-cap electrodes, coaxial with the trap and printed directly on the PCB holders (not shown [21]), are kept at a DC constant voltage. The particles are injected in low vacuum $\left(\sim 10^{-1}\right.$ mbar $)$ by means of electrospray ionization. A quadrupole mass filter [56] guides the particles from the input skimmer to the trapping region, which allows a better charge-to-mass ratio selection and an increased particle flux into the trap.

The linear Paul trap is characterized by 4 parameters, two of which relate to the actual trap geometry and two to the efficiency coefficients that quantify nonperfect quadratic potentials. The former are the distance between the center of the trap and the AC and DC electrodes, respectively where $r_{o}=1.1 \mathrm{~mm}$ and $z_{o}=3.5 \mathrm{~mm}$. The latter are $\eta=0.82$ which represents the quadrupolar coefficient of the multipole expansion of the AC pseudo-potential and $\kappa=0.086$ which is the quadratic coefficient of the DC potential at the trap center $[57,58]$. These are calculated with numerical simulations based on the finite element method (FEM). The Mathieu stability parameters are given by

$$
\begin{aligned}
& a_{x}=a_{y}=-\frac{1}{2} a_{z}=-\frac{q}{m} \frac{4 \kappa U_{o}}{z_{o}^{2} \omega_{d}^{2}}, \\
& q_{x}=-q_{y}=\frac{q}{m} \frac{2 \eta V_{o}}{r_{o}^{2} \omega_{d}^{2}}, q_{z}=0,
\end{aligned}
$$


where $U_{o}$ and $V_{o}$ are respectively the DC potential and the amplitude of the AC field, $q$ is the total electric charge, $m$ is the mass of the particle and $\omega_{d}$ is the $\mathrm{AC}$ drive frequency. Typical values for the DC voltage are from 50 to $150 \mathrm{~V}$, while for the AC, we have amplitudes between 100 and $300 \mathrm{~V}$ with frequencies from 1 to $5 \mathrm{kHz}$. A detailed characterization of the trap, its stability and the loading approach can be found in Ref. [21]. Using the stability parameters of Eq. (A1), we can write the Matheiu equation of motion for the particle position $u_{i}$ along the $i$ axis:

$$
\ddot{u}_{i}+\left[a_{i}+2 q_{i} \cos \left(\omega_{d} t\right)\right] \frac{\omega_{d}^{2}}{4} u_{i}=0 .
$$

To the lowest order in $a_{i}$ and $q_{i}$ solutions of Eq. (A3) are of the form

$$
u(t)=u_{o} \cos \left(\omega_{i} t\right)\left[1+\frac{q_{i}}{2} \cos \left(\omega_{d} t\right)\right],
$$

which describes a slow oscillation at $\omega_{i}$ called secular motion and a fast oscillation at $\omega_{d} \pm \omega_{i}$ called micromotion. If $q_{i} \lesssim$ 0.4 the two components are adiabatically separable, in this case, a pseudopotential approximation [59] provides a very good description of the dynamics, i.e., the secular motion is harmonic with frequencies given by $\omega_{i} \cong \omega_{d} / 2 \sqrt{a_{i}+q_{i}^{2} / 2}$. In our experiment, the low- $q$ condition is satisfied allowing us to describe the motion under stochastic thermal driving with the Langevin equation:

$$
\ddot{u}_{i}+\gamma \dot{u}_{i}+\omega_{i}^{2} u_{i}=F_{\mathrm{th}} / m,
$$

where $F_{\text {th }}$ is a delta-correlated force noise with spectral density $S_{F_{\mathrm{th}} F_{\mathrm{th}}}=2 k_{B} T m \gamma$; Eq. (A4) allow us to define the mechanical susceptibility as $\chi(\omega)=\left[m\left(\omega_{i}^{2}-\omega^{2}-\imath \omega \gamma\right)\right]^{-1}$ which has been used to fit the experimental PSD in Fig 2.

\section{APPENDIX B: DETECTION}

We exploit a simple detection scheme [38], where the trapped nanoparticle is illuminated with a $532 \mathrm{~nm}$ laser and the light scattered from it at $90^{\circ}$ is collected by a zoom objective lens and imaged onto a CMOS camera. The particle position is extracted by locating the coordinates of the brightest pixel. This simple approach allows real time acquisition of the particle motion in the plane defined by the field of view of the camera. The maximum sample rate ranges from 500 to $800 \mathrm{~Hz}$, which is sufficient for resolving the low secular frequencies in the trap. The laser beam waist is $250 \mu \mathrm{m}$ located near the trap center with a typical laser power of 40 mW.

\section{APPENDIX C: EFFECT OF VOLTAGE NOISE}

The most important contribution to the force noise acting on the nanoparticle is voltage noise from the AC electrodes. Its evaluation requires the knowledge of the electric field near the trap center which was obtained from finite element modeling simulations based on our trap voltages and geometry. With a voltage $V$ applied on both AC electrodes, and taking contributions up to the first order, we obtain

$$
\frac{E(x, y)}{V}=\left(\frac{1}{D}+\frac{\langle x\rangle}{D_{1}}\right) \check{x}+\left(\frac{1}{D}+\frac{\langle y\rangle}{D_{1}}\right) \check{y},
$$

where $\langle x\rangle$ and $\langle y\rangle$ are the mean particle positions, $D=2.3 \mathrm{~mm}$ and $D_{1}=1.5 \mu \mathrm{m}$. Assuming uncorrelated voltage noise and that the particle is at the trap center, the force noise PSD can then be evaluated by $S_{\mathrm{ff}}=\left(n_{\mathrm{ch}} e\right)^{2} S_{\mathrm{VV}} / D^{2}$ [60] which gives a voltage noise of $\sqrt{S_{\mathrm{VV}}} \simeq 10 \mu \mathrm{V} / \sqrt{\mathrm{Hz}}$, as in the main text. This value, however, is a rough estimation for several reasons: first, it assumes the particle is perfectly positioned in the trap center,i.e., $\langle x\rangle=\langle y\rangle=0$; variations of the mean position over the long experimental times could lead to deviations of the measured temperatures at low pressure (Fig. 6). Secondly, since the thermal induced displacement fluctuations along $x$ and $y$ are greater than $D_{1}$ the force noise will depend upon the instantaneous position of the particle leading to a stochastic term proportional to $V(t) x(t)$, which is the product between voltage and position fluctuations, i.e., a multiplicative noise. Finally, from geometrical considerations, the force noise on the particle due to voltage noise on the electrodes should be significantly lower along the $z$ axis (endcaps); this is clearly not the case and suggests a significant cross-coupling between degrees of freedom likely to be due to nonlinearity in the potential.

\section{APPENDIX D: COLLAPSE RATES}

In the dCSL model the collapse strength, $\eta_{\mathrm{dCSL}}$, for a spherical particle of radius $r$ and homogeneous density $\rho$ is given by [22]

$$
\begin{aligned}
\eta_{\mathrm{dCSL}}= & \frac{3 \lambda r_{C}^{2} m^{2}}{\left(1+\chi_{\mathrm{C}}\right) r^{4} m_{0}^{2}}\left[1-\frac{2 r_{C}^{2}\left(1+\chi_{\mathrm{C}}\right)^{2}}{r^{2}}\right. \\
& \left.+e^{-\frac{r^{2}}{r_{C}^{2}\left(1+\chi_{\mathrm{C}}\right)^{2}}}\left(1+\frac{2 r_{C}^{2}\left(1+\chi_{\mathrm{C}}\right)^{2}}{r^{2}}\right)\right],
\end{aligned}
$$

where $m$ is the total mass of the system, $m_{0}$ is the nucleon mass, $\chi_{\mathrm{C}}=\hbar^{2} /\left(8 m_{a} k_{B} T_{\mathrm{dCSL}} r_{C}^{2}\right)$, and $T_{\mathrm{dCSL}}$ is the thermalization temperature. In the limit of no dissipation, $T_{\mathrm{dCSL}} \rightarrow \infty$, we have $\chi_{\mathrm{C}} \rightarrow 0$, and thus one recovers the collapse strength of the standard CSL model, $\eta_{\mathrm{dCSL}} \rightarrow \eta_{\mathrm{CSL}}$. On the other hand, in case of strong dissipation when $a \ll r_{C}\left(1+\chi_{\mathrm{C}}\right)$, we obtain an approximate expression

$$
\eta_{\mathrm{dCSL}}=\frac{m m_{a} \lambda r_{C}}{2 a^{3} m_{0}^{2}\left(1+\chi_{\mathrm{C}}\right)^{2}} \min \left[1, \frac{r^{3}}{r_{C}^{3}\left(1+\chi_{\mathrm{C}}\right)^{3}}\right]
$$

where $m_{a}$ is the average mass of a nucleus ( $\simeq$ average atomic mass), and $a$ is the lattice constant [61]. Finally, the dissipation rate is given by $\gamma_{\mathrm{dCSL}}=\eta_{\mathrm{dCSL}} 4 r_{C}^{2} \chi_{\mathrm{C}}\left(1+\chi_{\mathrm{C}}\right) m_{a} / m$.

The calculation for the $\mathrm{dDP}$ model under the assumption of a homogeneous sphere can be carried out similarly as for the dCSL model. Specifically, we find the following collapse strength:

$$
\begin{aligned}
\eta_{\mathrm{dDP}}= & \frac{G m_{a}^{2}}{\sqrt{\pi} r^{6} \hbar}\left[\sqrt{\pi} r^{3} \operatorname{Erf}\left(\frac{r}{R_{0}\left(1+\chi_{0}\right)}\right)\right. \\
& +\left(1+\chi_{0}\right) R_{0}\left\{r^{2}\left(e^{-\frac{r^{2}}{\left(1+\chi_{0}\right)^{2} R_{0}^{2}}}-3\right)\right. \\
& \left.\left.+2\left(1+\chi_{0}\right)^{2} R_{0}^{2}\left(1-e^{-\frac{r^{2}}{\left(1+\chi_{0}\right)^{2} R_{0}^{2}}}\right)\right\}\right],
\end{aligned}
$$




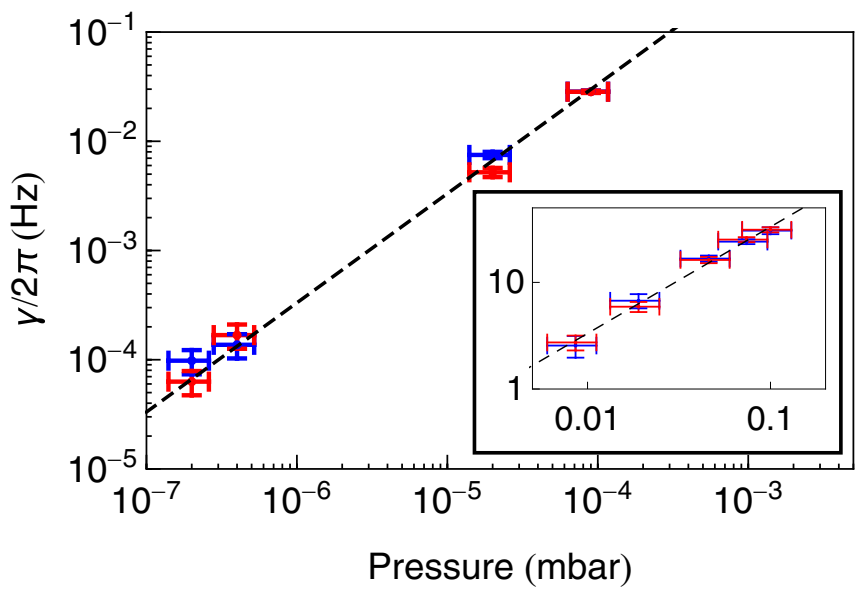

FIG. 8. Measured linewidths as a function of pressure along both $x$ (blue) and $z$ (red) axes obtained from the $R^{2}$ spectra. (Inset) Linewidth measured at high pressure by standard spectral analysis. The dashed line is a fit of the expected $1 / P_{g}$ behavior. Only the data in the inset have been used for the fit.

where $\chi_{0}=\hbar^{2} /\left(8 m_{a} k_{B} T_{\mathrm{dDP}} R_{0}^{2}\right)$, and $T_{\mathrm{dDP}}$ is the thermalization temperature. In the limit of no dissipation, $T_{\mathrm{dDP}} \rightarrow \infty$, we have $\chi_{\mathrm{dDP}} \rightarrow 0$, and the collapse strength of the standard DP model is recovered, $\eta_{\mathrm{dDP}} \rightarrow \eta_{\mathrm{DP}}$ [61]. Using a lattice model for the crystal structure we obtain an approximate formula for the case of strong dissipation, $a \ll R_{0}\left(1+\chi_{0}\right)$, given by

$$
\eta_{\mathrm{dDP}}=\frac{G m m_{a}}{6 \sqrt{\pi} a^{3} \hbar} \min \left[1, \frac{r^{3}}{R_{0}^{3}\left(1+\chi_{0}\right)^{3}}\right] .
$$

The damping rate for the dDP model is given by $\gamma_{\mathrm{dDP}}=$ $\eta_{\mathrm{dDP}} 4 R_{0}^{2} \chi_{0}\left(1+\chi_{0}\right) m_{a} / m$.

The CSL and DP family of models share many common features, among which the amplification of the collapse strength with the size of the system [26]. Such an amplification mechanism is supposed to accomplish a dual task: on the one hand, agreement with the quantum mechanical predictions for very small systems, and that classicality emerges for large systems [62]. The amplification mechanism has its origin in microscopic derivations where the center-ofmass dynamics of a bulk material is obtained starting from the dynamics of nucleons or nuclei. In the present case, this is reflected in geometrical factors $\eta_{\mathrm{dCSL}}$ and $\eta_{\mathrm{dDP}}$ that describe the effect of the intensive and extensive properties of the system on the center-of-mass dynamics. In the above formulas, we have chosen the nuclei as the building blocks of the models, motivated by considerations about gravity in the Newtonian regime [63].

However, one could also postulate that the center-of-mass motion is always in the single-particle form. In particular, one can require that the center-of-mass dynamics depends only on the total mass $m$ of the system, but not on other intensive or extensive properties of the bulk system. This latter choice is suggestive of a hypothetical underlying theory of spontaneous collapse models, which includes notions of Einstein's general relativity [64]. In this latter case, one has that the collapse strengths are given by $\eta_{\mathrm{dCSL}}=\frac{\lambda m^{2}}{2 m_{0}^{2} r_{C}^{2}\left(1+\chi_{\mathrm{C}}\right)^{5}}$ and $\eta_{\mathrm{dDP}}=\frac{G m^{2}}{6 \sqrt{\pi} \hbar R_{0}^{3}\left(1+\chi_{0}\right)^{3}}$, and the dissipation rates are obtained by replacing $m_{a}$ with $m$ and $a$ with $r$ in the corresponding formulas.

\section{APPENDIX E: LINEWIDTH MEASUREMENT}

In order to verify the accuracy of the $R^{2}$ method to measure the linewidth, we compare the low pressure data with the fit obtained exclusively with the high pressure measurements performed with the standard spectral method using the PSD. This is shown in Fig. 8 where the linewidths along the two monitored axes are fitted separately. All the low-pressure measurements are consistent with the expected behavior.
[1] P. F. Barker and M. N. Shneider, Cavity cooling of an optically trapped nanoparticle, Phys. Rev. A 81, 023826 (2010).

[2] D. E. Chang, C. A. Regal, S. B. Papp, D. J. Wilson, J. Ye, O. Painter, H. J. Kimble, and P. Zoller, Cavity opto-mechanics using an optically levitated nanosphere, Proc. Natl. Acad. Sci. USA 107, 1005 (2009).

[3] O. Romero-Isart, M. L. Juan, R. Quidant, and J. I. Cirac, Toward quantum superposition of living organisms, New J. Phys. 12, 033015 (2010).

[4] T. Li, S. Kheifets, and M. G. Raizen, Millikelvin cooling of an optically trapped microsphere in vacuum, Nat. Phys. 7, 527 (2011)

[5] J. Millen, P. Z. G. Fonseca, T. Mavrogordatos, T. S. Monteiro, and P. F. Barker, Cavity Cooling a Single Charged Levitated Nanosphere, Phys. Rev. Lett. 114, 123602 (2015).

[6] P. Z. G. Fonseca, E. B. Aranas, J. Millen, T. S. Monteiro, and P. F. Barker, Nonlinear Dynamics and Strong Cavity Cooling of Levitated Nanoparticles, Phys. Rev. Lett. 117, 173602 (2016).

[7] I. Alda, J. Berthelot, R. A. Rica, and R. Quidant, Trapping and manipulation of individual nanoparticles in a planar paul trap, Appl. Phys. Lett. 109, 163105 (2016).
[8] T. Delord, P. Huillery, L. Schwab, L. Nicolas, L. Lecordier, and G. Hétet, Ramsey Interferences and Spin Echoes from Electron Spins Inside a Levitating Macroscopic Particle, Phys. Rev. Lett. 121, 053602 (2018).

[9] C. C. Rusconi, V. Pöchhacker, K. Kustura, J. I. Cirac, and O. Romero-Isart, Quantum Spin Stabilized Magnetic Levitation, Phys. Rev. Lett. 119, 167202 (2017).

[10] B. R. Slezak, C. W. Lewandowski, J.-F. Hsu, and B. D’Urso, Cooling the motion of a silica microsphere in a magnetogravitational trap in ultra-high vacuum, New J. Phys. 20, 063028 (2018).

[11] E. Hebestreit, M. Frimmer, R. Reimann, and L. Novotny, Sensing Static Forces with Free-Falling Nanoparticles, Phys. Rev. Lett. 121, 063602 (2018).

[12] J. Bateman, S. Nimmrichter, K. Hornberger, and H. Ulbricht, Near-field interferometry of a free-falling nanoparticle from a point-like source, Nat. Commun. 5, 4788 (2014).

[13] O. Romero-Isart, A. C. Pflanzer, F. Blaser, R. Kaltenbaek, N. Kiesel, M. Aspelmeyer, and J. I. Cirac, Large Quantum Superpositions and Interference of Massive Nanometer-Sized Objects, Phys. Rev. Lett. 107, 020405 (2011). 
[14] A. A. Geraci, S. B. Papp, and J. Kitching, Short-Range Force Detection Using Optically Cooled Levitated Microspheres, Phys. Rev. Lett. 105, 101101 (2010).

[15] A. Arvanitaki and A. A. Geraci, Detecting High-Frequency Gravitational Waves with Optically Levitated Sensors, Phys. Rev. Lett. 110, 071105 (2013).

[16] D. C. Moore, A. D. Rider, and G. Gratta, Search for Millicharged Particles Using Optically Levitated Microspheres, Phys. Rev. Lett. 113, 251801 (2014).

[17] J. Gieseler, B. Deutsch, R. Quidant, and L. Novotny, Subkelvin Parametric Feedback Cooling of a Laser-Trapped Nanoparticle, Phys. Rev. Lett. 109, 103603 (2012).

[18] E. Hebestreit, M. Frimmer, R. Reimann, C. Dellago, F. Ricci, and L. Novotny, Calibration and energy measurement of optically levitated nanoparticle sensors, Rev. Sci. Instrum. 89, 033111 (2018).

[19] V. Jain, J. Gieseler, C. Moritz, C. Dellago, R. Quidant, and L. Novotny, Direct Measurement of Photon Recoil from a Levitated Nanoparticle, Phys. Rev. Lett. 116, 243601 (2016).

[20] J. Millen, T. Deesuwan, P. Barker, and J. Anders, Nanoscale temperature measurements using non-equilibrium brownian dynamics of a levitated nanosphere, Nat. Nanotechnol. 9, 425 (2014).

[21] N. P. Bullier, A. Pontin, and P. F. Barker, Characterisation of a charged particle levitated nano-oscillator, J. Phys. D: Appl. Phys. 53, 175302 (2019).

[22] J. Nobakht, M. Carlesso, S. Donadi, M. Paternostro, and A. Bassi, Unitary unraveling for the dissipative continuous spontaneous localization model: Application to optomechanical experiments, Phys. Rev. A 98, 042109 (2018).

[23] G. C. Ghirardi, P. Pearle, and A. Rimini, Markov processes in hilbert space and continuous spontaneous localization of systems of identical particles, Phys. Rev. A 42, 78 (1990).

[24] G. C. Ghirardi, R. Grassi, and F. Benatti, Describing the macroscopic world: Closing the circle within the dynamical reduction program, Found. Phys. 25, 5 (1995).

[25] A. Smirne and A. Bassi, Dissipative continuous spontaneous localization (CSL) model, Sci. Rep. 5, 12518 (2015).

[26] M. Bahrami, A. Smirne, and A. Bassi, Role of gravity in the collapse of a wave function: A probe into the diósi-penrose model, Phys. Rev. A 90, 062105 (2014).

[27] R. Penrose, On gravity's role in quantum state reduction, Gen. Relativ. Gravit. 28, 581 (1996).

[28] L. Diósi, Models for universal reduction of macroscopic quantum fluctuations, Phys. Rev. A 40, 1165 (1989).

[29] D. Goldwater, M. Paternostro, and P. F. Barker, Testing wavefunction-collapse models using parametric heating of a trapped nanosphere, Phys. Rev. A 94, 010104 (2016).

[30] A. Vinante, A. Pontin, M. Rashid, M. Toroš, P. F. Barker, and H. Ulbricht, Testing collapse models with levitated nanoparticles: Detection challenge, Phys. Rev. A 100, 012119 (2019).

[31] D. J. Berkeland, J. D. Miller, J. C. Bergquist et al., Minimization of ion micromotion in a paul trap, J. Appl. Phys. 83, 5025 (1998).

[32] P. S. Epstein, On the resistance experienced by spheres in their motion through gases, Phys. Rev. 23, 710 (1924).

[33] A. Cavalleri G. Ciani, R. Dolesi, M. Hueller, D. Nicolodi, D. Tombolato, S. Vitale, P. J. Wass, and W. J. Weber, Gas damping force noise on a macroscopic test body in an infinite gas reservoir, Phys. Lett. A 374, 3365 (2010).

[34] D. S. Bykov, P. Mestres, L. Dania, L. Schmöger, and T. E. Northup, Direct loading of nanoparticles under high vacuum into a paul trap for levitodynamical experiments, Appl. Phys. Lett. 115, 034101 (2019).

[35] A. Pontin, M. Bonaldi, A. Borrielli, F. S. Cataliotti, F. Marino, G. A. Prodi, E. Serra, and F. Marin, Detection of weak stochastic forces in a parametrically stabilized micro-optomechanical system, Phys. Rev. A 89, 023848 (2014).

[36] Y. Mishin and J. Hickman, Energy spectrum of a langevin oscillator, Phys. Rev. E 94, 062151 (2016).

[37] The first error is statistical, the second is systematic.

[38] N. P. Bullier, A. Pontin, and P. F. Barker, Super-resolution imaging of a low frequency levitated oscillator, Rev. Sci. Instrum. 90, 093201 (2019).

[39] L. Haiberger, D. Jäger, and S. Schiller, Fabrication and laser control of double-paddle silicon oscillators, Rev. Sci. Instrum. 76, 045106 (2005)

[40] E. Serra, M. Bonaldi, A. Borrielli, and L. Conti, Vibrational characterization of high-Q silicon resonators for investigating thermal noise statistical properties, International Conference on Noise and Vibration Engineering 2012, ISMA 2012, including USD 2012: International Conference on Uncertainty in Structure Dynamics, Vol. 4 (Katholieke Universiteit Leuven, 2012), pp. 3239-3245.

[41] D. F. McGuigan, C. C. Lam, R. Q. Gram, A. W. Hoffman, D. H Douglass, and H. W. Gutche , Measurements of the mechanical Q of single-crystal silicon at low temperatures, J. Low Temp. Phys. 30, 621 (1978).

[42] S. Wheaton, R. M. Gelfand, and R. Gordon, Probing the ramanactive acoustic vibrations of nanoparticles with extraordinary spectral resolution, Nat. Photonics 9, 68 (2014).

[43] G. C. Ghirardi, A. Rimini, and T. Weber, Unified dynamics for microscopic and macroscopic systems, Phys. Rev. D 34, 470 (1986).

[44] R. Penrose, Quantum computation, entanglement and state reduction, Philos. Trans. R. Soc. London A 356, 1927 (1998).

[45] A. Vinante, M. Bahrami, A. Bassi, O. Usenko, G. Wijts, and T. H. Oosterkamp, Upper Bounds on Spontaneous WaveFunction Collapse Models Using Millikelvin-Cooled Nanocantilevers, Phys. Rev. Lett. 116, 090402 (2016).

[46] A. Vinante, R. Mezzena, P. Falferi, M. Carlesso, and A. Bassi, Improved Noninterferometric Test of Collapse Models using Ultracold Cantilevers, Phys. Rev. Lett. 119, 110401 (2017).

[47] M. Armano et al., Beyond the Required Lisa Free-Fall Performance: New Lisa Pathfinder Results Down to $20 \mu \mathrm{Hz}$, Phys. Rev. Lett. 120, 061101 (2018).

[48] M. Carlesso, A. Bassi, P. Falferi, and A. Vinante, Experimental bounds on collapse models from gravitational wave detectors, Phys. Rev. D 94, 124036 (2016).

[49] M. Carlesso, M. Paternostro, H. Ulbricht, A. Vinante, and A. Bassi, Non-interferometric test of the continuous spontaneous localization model based on rotational optomechanics, New J. Phys. 20, 083022 (2018).

[50] M. Toroš, G. Gasbarri, and A. Bassi, Colored and dissipative continuous spontaneous localization model and bounds from matter-wave interferometry, Phys. Lett. A 381, 3921 (2017). 
[51] M. Toroš and A. Bassi, Bounds on quantum collapse models from matter-wave interferometry: calculational details, J. Phys. A: Math. Theor. 51, 115302 (2018).

[52] S. L. Adler, Lower and upper bounds on CSL parameters from latent image formation and IGM heating, J. Phys. A: Math. Theor. 40, 2935 (2007).

[53] S. L. Adler, Lower and upper bounds on CSL parameters from latent image formation and IGM rheating, J. Phys. A: Math. Theor. 40, 13501 (2007).

[54] S. W. Hawking, Black hole explosions? Nature (London) 248, 30 (1974).

[55] S. W. Hawking, Particle creation by black holes, Commun. Math. Phys. 43, 199 (1975).

[56] R. E. March, Quadrupole ion traps, Mass Spectrom. Rev. 28, 961 (2009).

[57] R. R. A. Syms, T. J. Tate, M. M. Ahmad, and S. Taylor, Design of a microengineered electrostatic quadrupole lens, IEEE Trans. Electron Devices 45, 2304 (1998).
[58] M. J. Madsen, W. K. Hensinger, D. Stick, J. A. Rabchuk, and C. Monroe, Planar ion trap geometry for microfabrication, Appl. Phys. B 78, 639 (2004).

[59] P. K. Ghosh, Ion Traps, International Series of Monographs on Physics (Oxford University Press, 1996).

[60] L. Deslauriers, S. Olmschenk, D. Stick, W. K. Hensinger, J. Sterk, and C. Monroe, Scaling and Suppression of Anomalous Heating in Ion Traps, Phys. Rev. Lett. 97, 103007 (2006).

[61] S. Nimmrichter, K. Hornberger, and K. Hammerer, Optomechanical Sensing of Spontaneous Wave-Function Collapse, Phys. Rev. Lett. 113, 020405 (2014).

[62] S. Nimmrichter and K. Hornberger, Macroscopicity of Mechanical Quantum Superposition States, Phys. Rev. Lett. 110, 160403 (2013).

[63] A. Tilloy and L. Diósi, Principle of least decoherence for newtonian semiclassical gravity, Phys. Rev. D 96, 104045 (2017).

[64] R. Penrose, On the gravitization of quantum mechanics 1: Quantum state reduction, Found. Phys. 44, 557 (2014). 